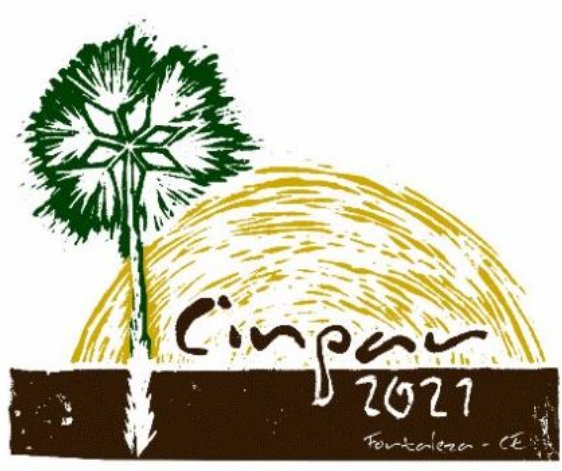

XVII Congresso Internacional sobre Patologia e

Reabilitação das Construções

XVII Congreso Internacional sobre Patología y Rehabilitación de las Construcciones

XVII International Conference on Pathology and Constructions Rehabilitation

FORTALEZA (Brasil), 3 a 5 de junho de 2021

https://doi.org/10.4322/CINPAR.2021.086

\title{
Estudo Sobre Manchamento de Engobe em Revestimento Cerâmico
}

\section{Study on engobe staining in ceramic coating}

\author{
Francisco Gabriel Santos SILVA ${ }^{1}$, Luanne Bastos de Britto BARBOSA ${ }^{2}$, Vinícius Almeida COELHO ${ }^{3}$ \\ ${ }^{1}$ Universidade Federal da Bahia, Escola Politécnica - Departamento de Construção e Estruturas, Brasil, \\ fgabriel.ufba@gmail.com \\ ${ }^{2}$ Universidade Federal da Bahia, Escola Politécnica - Programa de Pós-Graduação em Engenharia Civil (PPEC), Brasil, \\ lubastosb@gmail.com \\ ${ }^{3}$ Universidade Federal da Bahia, Escola Politécnica - Programa de Pós-Graduação em Engenharia Civil (PPEC), Brasil, \\ mrvoelho@gmail.com
}

Resumo: O revestimento cerâmico é um dos produtos mais utilizados para acabamento na construção civil. O qual tem a finalidade de auxiliar na proteção e durabilidade da edificação, podendo ser empregado para ambientes internos e externos, pisos, paredes e em locais com baixo ou alto tráfego. $O$ sistema de revestimento é susceptível à diversos tipos de manifestações patológicas, entre elas a patologia mancha d'água. Diante disso, este artigo apresenta um estudo de caso sobre o manchamento do piso cerâmico de uma unidade educacional da cidade de Itapetinga - BA. Foram coletadas amostras do piso manchado e do piso estocado, na qual se realizou ensaios de caracterização física (resistência ao manchamento, resistência ao ataque químico, absorção de água, pHmetria e perda de brilho superficial) e caracterização química através dos ensaios de Fluorescência de Raios X (FRX) e Difratometria de raios X (DRX). Foi possível concluir que a ocorrência das manchas não foi na superfície do verniz e sim na região do engobe. Na qual a sua ocorrência predominante se dá em cerâmicas porosas, principalmente classe Bllb. A patologia mancha d'água culmina na alteração da tonalidade da cerâmica quando ocorre a absorção de água pelo suporte cerâmico.

Palavras-chave: Patologias dos revestimentos; Piso cerâmico; Revestimento cerâmico.

\begin{abstract}
Ceramic tiles are one of the most used products for finishing in civil construction. It assists in the protection and durability of the building and can be used for indoor and outdoor environments, on floors, walls and in places with low or high traffic. The coating system is susceptible to several types of pathological manifestations, among them the water stain pathology. Therefore, this article presents a case study on the staining of the ceramic floor of an educational unit in the city of Itapetinga - BA. Samples of the stained floor and the stocked floor were collected, in which physical characterization tests (resistance to staining, resistance to chemical attack, water absorption, $\mathrm{pH}$-metry and loss of surface brightness) and chemical characterization through the tests of Fluorescence X-ray (FRX) and X-ray diffractometry (XRD). It was possible to conclude that the stains did not occur on the varnish surface, but in the engobe region. in which its predominant occurrence occurs in porous ceramics, mainly class Bllb. The water stain pathology culminates in the alteration of the ceramic tonality when water absorption by the ceramic support occurs.
\end{abstract}

Keywords: Coatings Pathologies; Ceramic floor; Ceramic tiles. 


\section{Introdução}

A patologia de manchamento na região do engobe é amplamente conhecida e estudada na comunidade científica (MELCHIADES et al., 2000, 2002, 2003; MONTE, 2008; PEREZ, 1991), na qual a sua ocorrência predominante se dá em cerâmicas porosas, principalmente classe Bllb. $O$ engobe é uma cobertura intermediária aplicada no corpo cerâmico cru ou queimado. É constituído por uma mistura de argilas, caulins, materiais não plásticos como quartzo, feldspatos, sienitas, fritas fundentes, etc e, algumas vezes, corantes cerâmicos (Figura 1).

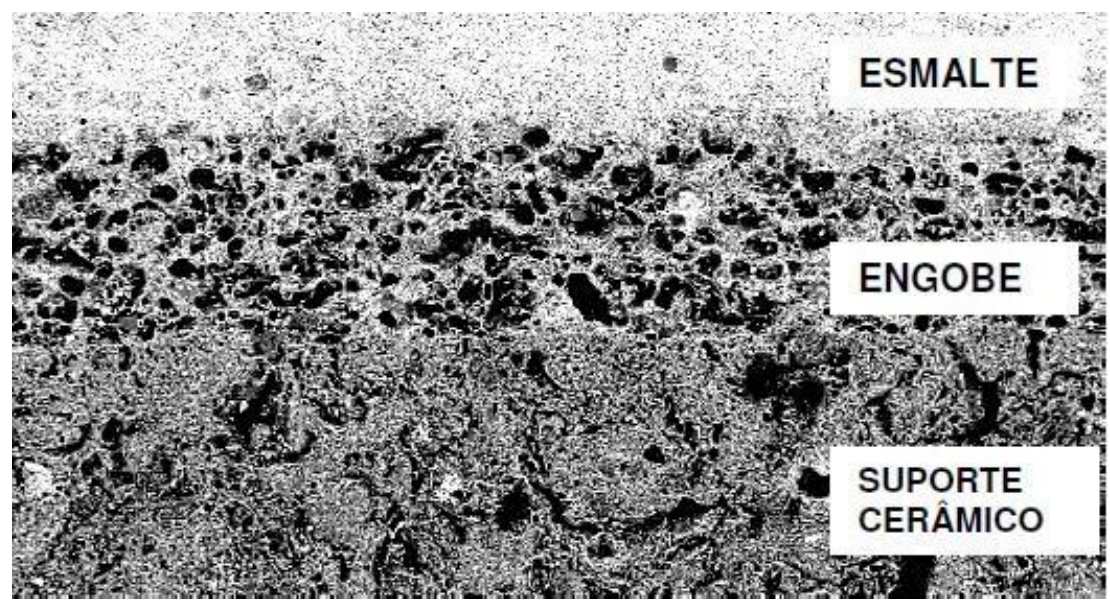

Figura 1 - Imagem ilustrativa das camadas que constituem os revestimentos cerâmicos esmaltados, obtidas por MEV (Fonte: MONTE, 2008)

Conforme análise estatística dos dados coletados pelo Centro Cerâmico do Brasil (CCB) mostrada na Figura $2,50,8 \%$ dos produtos que apresentaram mancha d'água foram da classe Bllb, seguida da BIII, com $33,3 \%$. Segundo a norma ISO 13.006 e MELCHIADES et al. (2002) esses índices podem ser decorrentes de falhas de impermeabilização da região do engobe no processo de fabricação da peça, na qual o mesmo apresenta-se com elevada porosidade (MELCHIADES et al., 2000, 2002, 2003; MONTE, 2008; PEREZ, 1991).

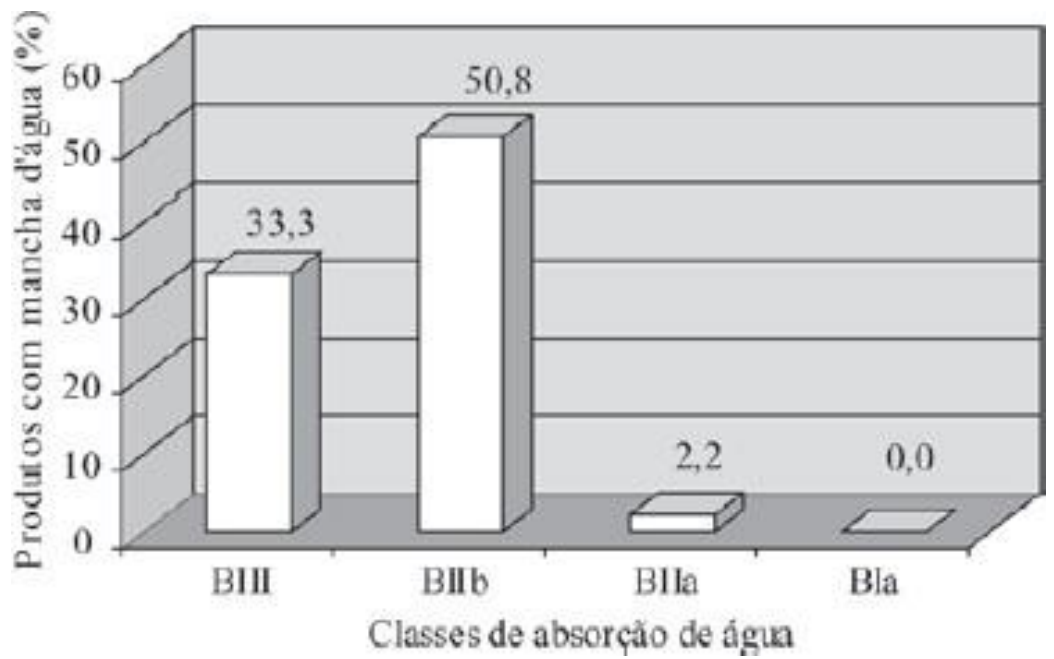

Figura 2 - Efeito da classe de absorção sobre o aparecimento da mancha d'água

Segundo Melchiades et al. (2000, 2002 e 2003), as causas da mancha d'água estão ligadas às características do esmalte, engobe, suporte e processamento. Industrialmente, a principal maneira de controlar a mancha 
d'água é através do controle das características do engobe (PEREZ, 1991), ainda segundo o autor, os engobes impermeáveis não apresentam alteração de sua cor mesmo quando ocorre a absorção de água por parte do suporte e, por serem opacos, impedem que a alteração de cor sofrida pelo suporte seja vista a partir da face esmaltada do revestimento.

A obtenção de engobes impermeáveis, entretanto, depende fundamentalmente: a) da composição e das características físicas e químicas de cada componente; b) das condições de fabricação do engobe; c) das condições de aplicação e d) das condições de queima. Além disso, é importante ressaltar a importância da compatibilidade das propriedades do engobe com as do suporte, esmalte e as condições de fabricação, ou seja, é preciso desenvolver engobes que sejam impermeáveis sem se esquecer dos demais requisitos para um bom engobe.

Percebe-se que nos estudos científicos citados (MELCHIADES et al., 2000, 2002, 2003; MONTE, 2008; PEREZ, 1991), a patologia de engobe está intimamente ligada à posoridade adquirida no processo de fabricação da cerâmica, que deve ser apropriada para o uso de matrizes cimentícias hidratadas, pois o contato do revestimento cerâmico com a água, seja durante o assentamento, seja durante o uso, é praticamente impossível de ser evitado. Sendo assim, a eliminação desta patologia só poderá ocorrer através da produção de placas cerâmicas que não mudem de tonalidade quando o suporte entra em contato com água.

\section{Materiais e métodos}

\subsection{Material}

O material utilizado nesta pesquisa foi a Placa Cerâmica Esmaltada, com classe A, PEI: 4. Formato: 45 x 45 $\mathrm{cm}$. Seu processo de fabricação é tipo prensado, com classe de absorção Bllb (Figura 3).

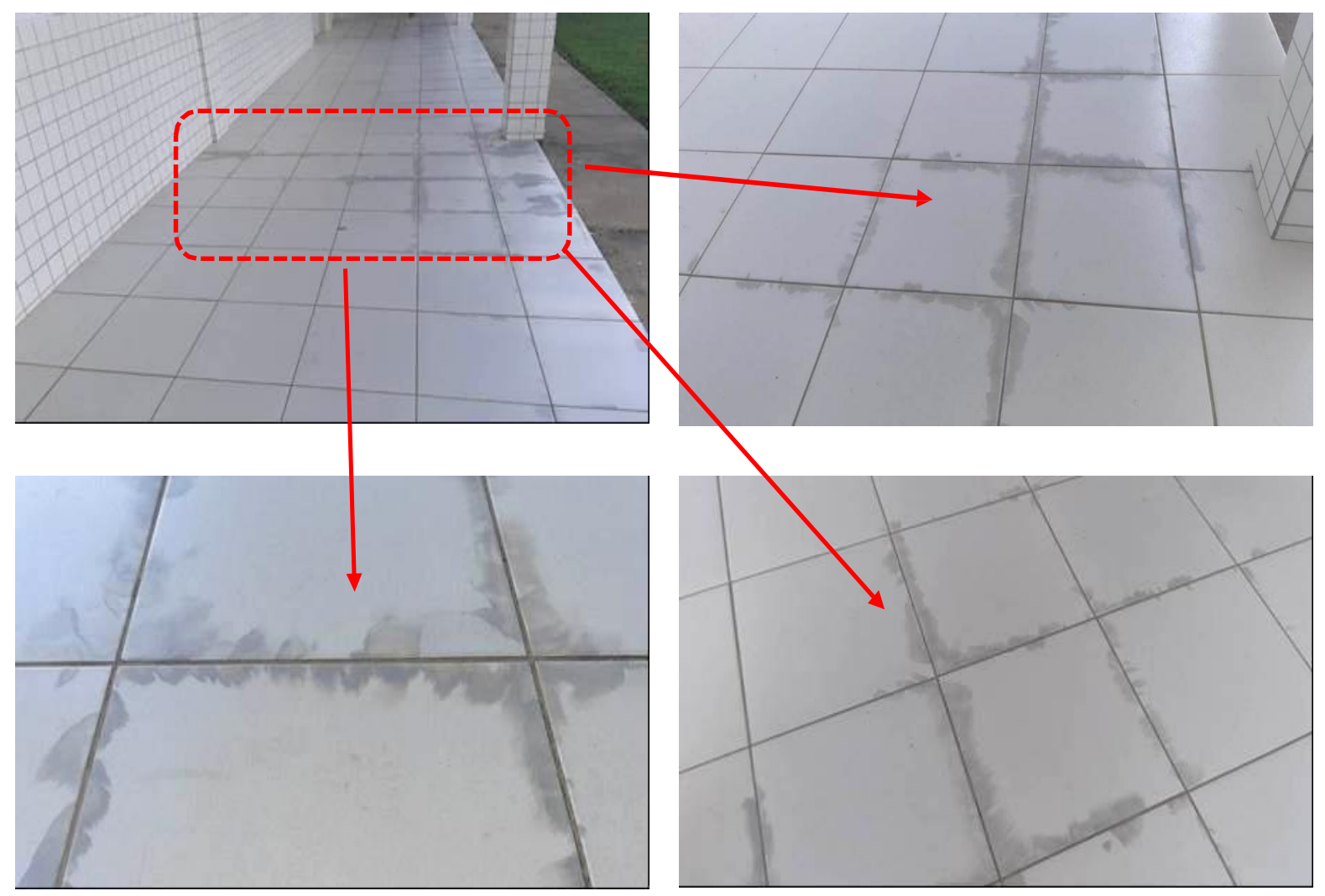

Figura 3 - Piso cerâmico com mancha d'água 


\subsection{Métodos}

\subsection{1 - Análise de caracterização física da cerâmica}

Foram realizados no laboratório de ensaios em materiais de construção (LEMC) do SENAI/CIMATEC em Salvador/BA. Os ensaios de caracterização estabelecidos pela NBR 13818: Resistência ao manchamento, Resistência ao ataque químico e absorção de água.

Quadro 1 - Descrição e classificação normativa dos ensaios de acordo à NBR 13818

\begin{tabular}{|c|c|c|}
\hline Ensaio & $\begin{array}{l}\text { Classificação } \\
\text { normativa }\end{array}$ & Descrição \\
\hline \multirow{4}{*}{$\begin{array}{l}\text { Resistência ao ataque } \\
\text { químico }\end{array}$} & GA & $\begin{array}{l}\text { Efeitos não visíveis após o tempo de contato ( } 24 \text { horas) } \\
\text { com a solução: elevada resistência química. }\end{array}$ \\
\hline & GLA & $\begin{array}{c}\text { Efeitos não visíveis após o tempo de contato (96 horas) } \\
\text { com a solução de baixa concentração: elevada resistência } \\
\text { química. }\end{array}$ \\
\hline & GHA & $\begin{array}{c}\text { Efeitos não visíveis após o tempo de contato (96 horas) } \\
\text { com a solução de alta concentração: elevada resistência } \\
\text { química }\end{array}$ \\
\hline & GLB & Mudança acentuada no aspecto ( 24 horas) com a solução \\
\hline \multirow{2}{*}{$\begin{array}{l}\text { Resistência ao } \\
\text { manchamento }\end{array}$} & CLASSE 3 & $\begin{array}{l}\text { Mancha removida removível com produto de limpeza } \\
\text { forte - saponáceo (média limpabilidade). }\end{array}$ \\
\hline & CLASSE 5 & $\begin{array}{l}\text { Mancha removida com a utilização de água quente: } \\
\text { máxima facilidade de remoção da mancha (alta } \\
\text { limpabilidade). }\end{array}$ \\
\hline Absorção de Água & CLASSE BIIb & $\begin{array}{l}\text { Cerâmica produzida de forma prensada, semiporosa, com } \\
\text { absorção de água entre } 6 \text { e } 10 \% .\end{array}$ \\
\hline
\end{tabular}

\subsection{2 - Análise de pHmetria e perda de brilho na superfície do esmalte}

Foi realizada a avaliação de pHmetria com solução alcoólica de Fenolftaleína para identificação de extrato lixiviado de hidróxido de cálcio do rejunte e produtos lixiviados da ação de enzimas dos supostos fungos desenvolvidos na superfície do esmalte. Além disso, foi realizada a avaliação de perda de brilho na superfície do esmalte utilizando luz refletida.

\subsection{3 - Análise química}

As análises química e mineralógica foram realizadas no Laboratório de Catálise no Instituto de Química na Universidade Federal da Bahia (UFBA), em Salvador/Ba. A análise elementar foi realizada num espectrofotômetro de fluorescência de raios $X$ com variação de comprimento de onda - WD-FRX, utilizando o equipamento $\mathrm{S} 8 \mathrm{Tiger}$ - Bruker. As amostras foram preparadas pelo método de prensagem sobre uma base de ácido bórico, e analisadas em alto vácuo utilizando o método quali-quante. Os resultados estão expressos em percentagem (\%), quando a concentração dos elementos está muito baixa, utiliza-se a unidade parte por milhão (ppm) que equivale a $\mathrm{mg} / \mathrm{Kg}$.

As fases cristalinas foram analisadas utilizando a técnica de difratometria de raios $X$, utilizando o equipamento XRD-6000, Shimadzu, operando com uma radiação monocromática CuK $\alpha=1,54056$ a 40kV, 30 $\mathrm{mA}, \lambda=5-80 \AA$, numa velocidade de $2^{\circ} \mathrm{min}-1$. A radiação foi colimada utilizando-se fendas de $1^{\circ}$ para 
divergência, $1^{\circ}$ para espelhamento e $0,3 \mathrm{~mm}$ para recepção. Os perfis de difração de raios-X das amostras foram identificados utilizando software X' Pert HighScore Plus.

\section{Resultados e discussões}

\subsection{Caracterização Fisica}

Os resultados da análise físico química realizada encontram-se no Quadro 2. Pode-se concluir que a classificação da cerâmica é Bllb (prensada, semiporosa) conforme especificado pelo fabricante e a resistência ao manchamento e ataque químico mostram que ela atende aos parâmetros normativos da NBR 13818, evidenciando assim que as manchas não são provenientes de produtos de limpeza sobre a superfície do verniz.

Quadro 2 - Resultado dos Ensaios físico-químicos

\begin{tabular}{|c|c|c|c|c|}
\hline Item & Ensaio & $\begin{array}{c}\text { Classificação } \\
\text { normativa }\end{array}$ & $\begin{array}{c}\text { Classificação } \\
\text { definida no } \\
\text { ensaio }\end{array}$ & Método \\
\hline 1.1 & $\begin{array}{c}\text { Resistência ao ataque } \\
\text { químico }\end{array}$ & GB & GA/GLA/GHA & NBR 13818 \\
\hline 1.2 & $\begin{array}{c}\text { Resistência ao } \\
\text { manchamento }\end{array}$ & $\geq 3$ & $\mathbf{5}$ & NBR 13818 \\
\hline 1.3 & Absorção de Água & Bllb & Bllb & NBR 13818 \\
\hline
\end{tabular}

\subsection{Phmetria e Perda de Brilho na Superficie do Esmalte}

Foi realizado numa amostra da cerâmica manchada o teste de pHmetria com solução alcoólica de Fenolftaleína para a identificação de vestígios de hidróxido de cálcio ou outros matéria alcalinos na superfície do verniz (Figura 4), na qual não foi identificada a presença de material alcalino (ausência de manchas rosas), evidenciando que não há depósitos de extratos lixiviados na superfície do verniz.

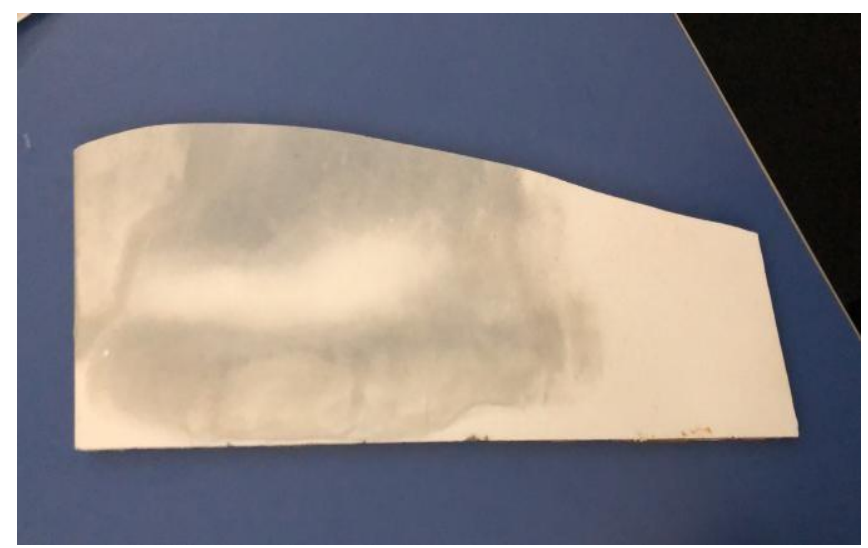

Figura 4 - Teste de pHmetria

No que se refere ao teste de perda de brilho, considerando que se há depósitos de extratos lixiviados e/ou enzimas de microrganismos e/ou outros materiais com capacidade de manchar o verniz perderia o brilho. Esse teste foi feito com luz refletida em laboratório (Figura 5a) e em ambiente externo com luz solar (Figura $5 b$ e Figura $5 c)$. 

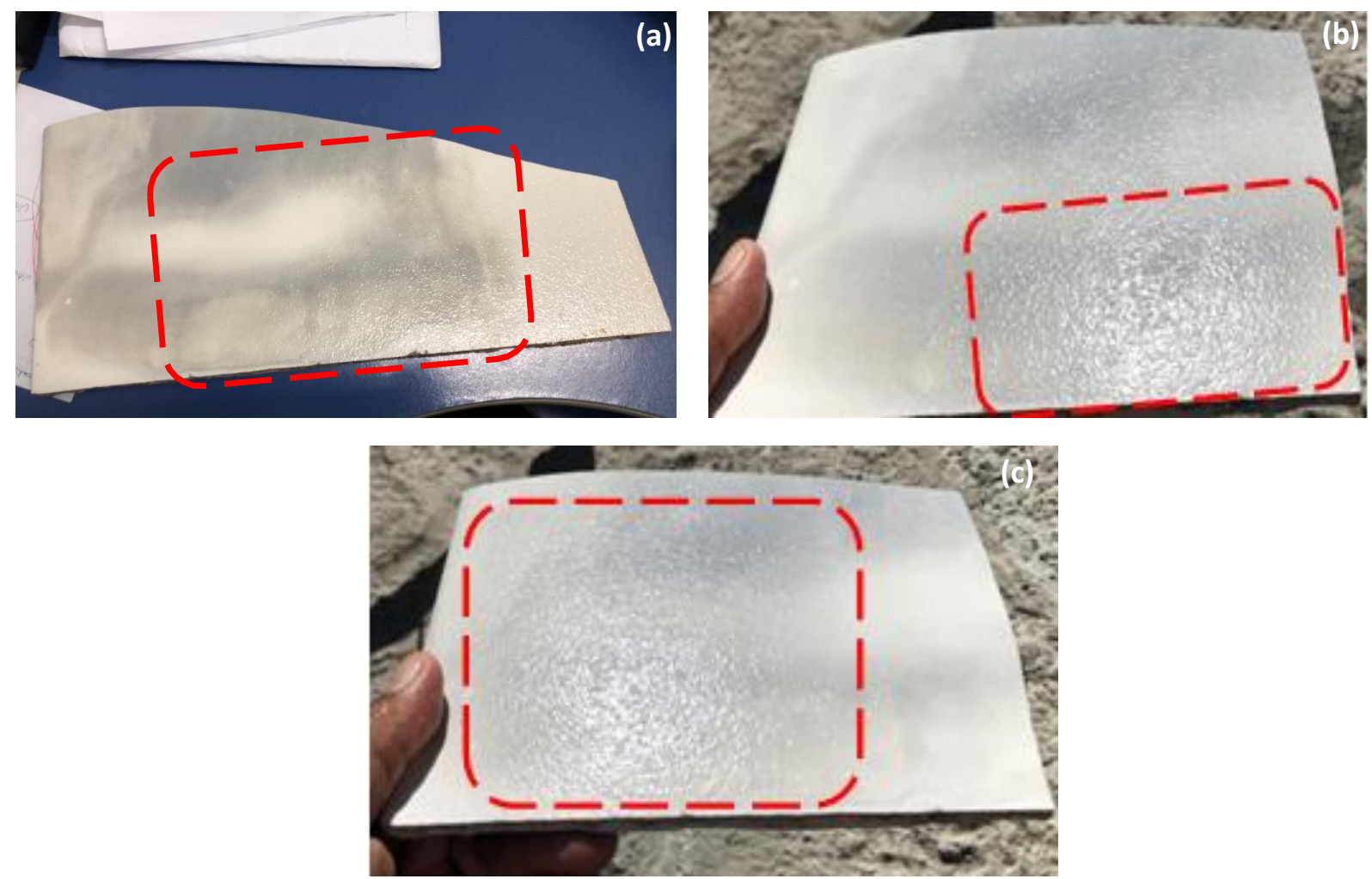

Figura 5 - Teste de brilho

Nas Figuras $5 a, 5 b$ e $5 c$ foi possível verificar que o verniz não perdeu o brilho, indicando que as manchas escurecidas não estão ocorrendo na superfície do verniz e sim sob essa superfície, na região conhecida como engobe, se tratando, portanto, de um defeito de fabricação da cerâmica com falta ou falha da impermeabilização da região do engobe.

\subsection{Análise química por FRX}

A análise elementar feita por FRX (Quadro 3) realizada na superfície do esmalte nas duas peças avaliadas, com mancha e sem mancha), mostram que a composição química de ambas as amostras são a mesma, não havendo variação expressiva dentro da margem de erro estatístico do equipamento, os principais elementos químicos, grifados em vermelho) tem praticamente o mesmo teor percentual. Isso reforça a análise anterior de que as manchas não estão ocorrendo na superfície do verniz e sim na região do engobe, pois se hovesse qualquer manchamento na superfície do verniz a composição química teria alteração percentual fora da margem de erro estatístico.

\subsection{Análise de DRX}

A análise elementar feita por DRX na superfície do verniz das duas amostras, íntegra e com mancha. Foi verificado que os difratogramas (Figura 5) das duas amostras têm o mesmo perfil cristalográfico, nos quais os principais produtos identificados, como mostrados no Quadro 4, são exatamente os mesmos, confirmando todas as análises anteriores. Ou seja, não foram detectadas manchas na superfície do verniz, e sim, na região do engobe 
Quadro 3 - Resultado dos ensaios de FRX

\begin{tabular}{|c|c|c|c|c|c|c|}
\hline \multirow{2}{*}{$\begin{array}{l}\text { Amostra/ } \\
\text { Elementos }\end{array}$} & \multicolumn{3}{|c|}{ Cerâmica íntegra } & \multicolumn{3}{|c|}{ Cerâmica com mancha } \\
\hline & Teor (\%) & $\begin{array}{l}\text { Erro } \\
(\%)\end{array}$ & $\begin{array}{c}\text { LLD } \\
\text { (ppm) }\end{array}$ & Teor (\%) & $\begin{array}{l}\text { Erro } \\
(\%)\end{array}$ & $\begin{array}{c}\text { LLD } \\
\text { (ppm) }\end{array}$ \\
\hline $\mathrm{Fe}_{2} \mathrm{O}_{3}$ & 0.21 & 1.14 & 48.8 & 0.24 & 1.07 & 43.5 \\
\hline $\mathrm{SO}_{3}$ & 0.05 & 6.71 & 145.7 & 0.08 & 5.21 & 126.7 \\
\hline $\mathrm{SiO}_{2}$ & 60.30 & 0.21 & 588.3 & 64.72 & 0.20 & 564.1 \\
\hline $\mathrm{Cl}$ & 0.04 & 6.40 & 77.7 & 0.04 & 6.37 & 75.7 \\
\hline MnO & 0.02 & 6.74 & 46.1 & 0.02 & 5.37 & 45.0 \\
\hline $\mathrm{CaO}$ & 13.54 & 0.29 & 419.2 & 13.13 & 0.29 & 393.9 \\
\hline $\mathrm{Al}_{2} \mathrm{O}_{3}$ & 12.72 & 0.44 & 242.6 & 11.83 & 0.45 & 243.7 \\
\hline MgO & 1.10 & 1.53 & 433.0 & 1.60 & 1.20 & 419.2 \\
\hline $\mathrm{Na}_{2} \mathrm{O}$ & 2.96 & 1.12 & $0.20 \%$ & 3.22 & 1.14 & $0.14 \%$ \\
\hline $\mathrm{BaO}$ & 0.17 & 4.62 & 236.2 & 0.14 & 5.09 & 220.3 \\
\hline $\mathrm{TiO}_{2}$ & 0.05 & 6.38 & 111.0 & 0.05 & 6.22 & 101.7 \\
\hline $\mathrm{K}_{2} \mathrm{O}$ & 3.16 & 0.56 & 74.9 & 2.65 & 0.61 & 66.0 \\
\hline $\mathbf{P}_{\mathbf{2}} \mathrm{O}_{5}$ & 0.14 & 4.85 & 206.9 & 0.23 & 3.75 & 148.4 \\
\hline $\mathrm{ZrO}_{2}$ & 1.09 & 0.17 & 73.7 & 0.30 & 0.30 & 59.0 \\
\hline SrO & 0.06 & 0.81 & 14.6 & 0.05 & 0.90 & 12.1 \\
\hline $\mathrm{ZnO}$ & 3.97 & 0.14 & 81.5 & 1.55 & 0.22 & 74.9 \\
\hline $\mathrm{Rb}_{2} \mathrm{O}$ & 0.01 & 2.24 & 16.3 & 0.01 & 2.03 & 13.4 \\
\hline PbO & 0.37 & 0.58 & 44.5 & 0.15 & 0.93 & 38.4 \\
\hline $\mathrm{HfO}_{2}$ & 0.03 & 3.56 & 136.3 & 96 ppm & 9.26 & 99.7 \\
\hline $\mathrm{CuO}$ & $28 \mathrm{ppm}$ & 18.10 & 25.0 & - & - & - \\
\hline $\mathrm{ThO}_{2}$ & 18 ppm & 6,37 & 71.4 & - & - & - \\
\hline 0 & 45.02 & 2,27 & $1.23 \%$ & 46.38 & 2.19 & $1.06 \%$ \\
\hline
\end{tabular}

Quadro 4 - Principais fases identificadas nas amostras íntegra e manchada

\begin{tabular}{|c|c|c|c|c|}
\hline \multirow{2}{*}{ Amostra } & $\begin{array}{c}\text { Cód. de } \\
\text { referência }\end{array}$ & Escore & $\begin{array}{c}\text { Nome do } \\
\text { composto }\end{array}$ & Fórmula química \\
\hline \multirow{2}{*}{ Íntegra } & $01-089-8938$ & 68 & Alfa Quartzo & $\mathrm{SiO}_{2}$ \\
\cline { 2 - 5 } & $01-073-1110$ & 33 & Wollastonita & $\mathrm{CaSiO}_{3}$ \\
\hline \multirow{2}{*}{ Manchada } & $01-089-8937$ & 64 & Alfa Quartzo & $\mathrm{SiO}_{2}$ \\
\cline { 2 - 5 } & $01-075-1396$ & 32 & Wollastonita & $\mathrm{CaSiO}_{3}$ \\
\hline
\end{tabular}



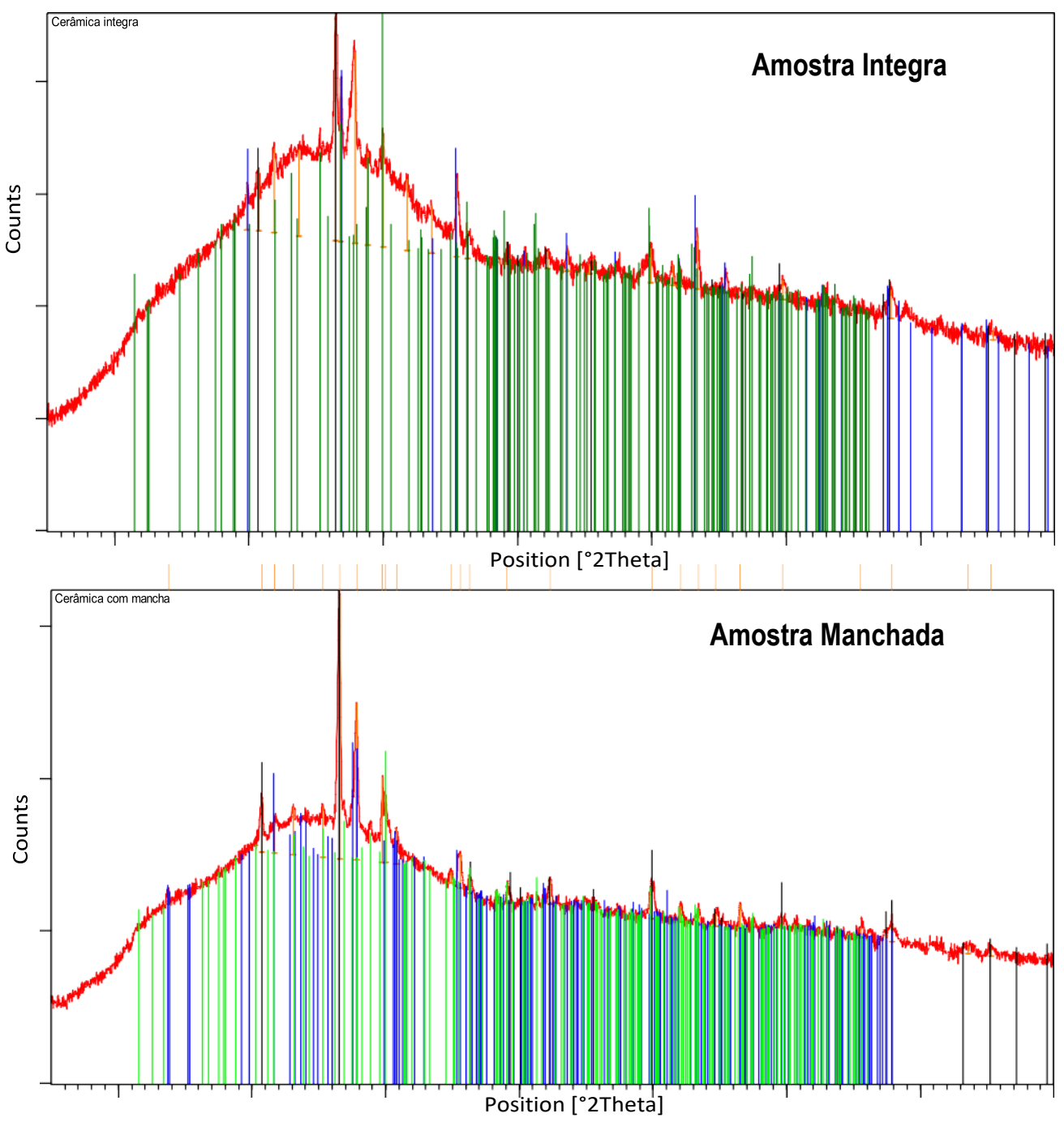

Figura 5 - Difratogramas das amostras integra e com mancha

\section{Conclusões}

Diante das análises realizadas nas duas amostras de cerâmica, com mancha e sem mancha, foi diagnosticado que a ocorrência das manchas se dá na região do engobe.

A patologia mancha d'água é um problema típico da indústria brasileira, que para baratear custo do produto e aumentar a produtividade, utiliza espessuras cada vez menores de engobe e ciclos de queima muito rápidos. Ainda segundo a literatura técnica e científica, os engobes impermeáveis não apresentam alteração de sua tonalidade quando ocorre a absorção de água pelo suporte cerâmico e, por serem, opacos, não permitem que a variação de cor sofrida pelo suporte seja visualizada a partir da superfície esmaltada da placa cerâmica.

Diante do exposto, e com base nos resultados dos ensaios realizados conclui que a causa da patologia de manchamento encontrado está relacionada à falha/falta de impermeabilização da região do engobe, sendo, portanto, de responsabilidade do fabricante das peças cerâmicas e não da empresa construtora que executou o piso. 


\section{Agradecimentos}

Agradecemos à Fundação de Amparo à Pesquisa do Estado da Bahia (FAPESB) pelo apoio financeiro e incentivo à pesquisa e a todas as instituições parceiras envolvidas no desenvolvimento desta pesquisa.

\section{Referências Bibliográficas}

ABNT. Associação Brasileira De Normas Técnicas. (1997). NBR 13818: Placas cerâmicas. Nov. 1997, p. 2.

Melchiades, F. G.; Silva, L. L.; Romachelli, J. C.; Boschi, A. O. A mancha dágua em revestimentos cerâmicos: contribuição para o desenvolvimento de um método de medida. Cerâmica Industrial, v. 4, n. 5, p. 26-30, 2000.

Melchiades, F. G.; Silva, L. L.; Silva, V. A.; Romachelli, J. C.; Vargas, D. D. T.; Boschi, A. O. (2002). Sobre engobes e a mancha d'água. Cerâmica Industrial., 7 (4), 31-39.

Melchiades, F. G.; Romachelli, J. C.; Boschi, A. O. (2003). A mancha d'água de revestimentos cerâmicos: defeito ou característica? Cerâmica Industrial, 8 (4), 7-10.

Monte, M. V. C.; Lima, N. B.; Menegazzo, A. P. M. (2008). Avaliação de engobes no aparecimento da mancha de Água em revestimentos cerâmicos. Dissertação de Mestrado, IPEN.

Perez, E.A. (1991). Apuntes de esmaltes y colores cerâmicos. Instituto de Formación Profissional no. 2 de Castellón, Generalitat Valenciana. 